\title{
NOTE ON A THEOREM OF KAKUTANI
}

\section{H. D. BRUNK}

1. Introduction. Limit theorems of probability have attracted much attention for a number of years. For a wide class of limit theorems, the language and methods of infinite product measure spaces have proved to be appropriate. It is the purpose of this note to discuss the application of a recent theorem of Kakutani on the equivalence of infinite product measures to the problem of extending known sufficient conditions for such theorems.

The distribution function of each of a sequence of independent real-valued random variables generates a measure on $R_{1}$, the space of real numbers. These measures determine a direct infinite product measure on the product space $R_{1} \times R_{1} \times \cdots$ Kakutani's theorem gives necessary and sufficient conditions for the equivalence of two direct infinite product measures. If the infinite product measures associated with two sequences of random variables are equivalent, then they both obey, or both fail to obey, any of a wide class of limit theorems. Thus if one sequence of independent random variables satisfies known sufficient conditions for such a limit theorem, and the two associated product measures satisfy Kakutani's conditions for equivalence, then the other sequence also obeys the limit theorem. In this way Kakutani's theorem provides a device for extending known sufficient conditions. In its present form, it does not contain a certain well known device of this type. Indeed, for this purpose, full equivalence of the product measures is not in general necessary. However, it is possible to derive, from Kakutani's conditions, conditions apparently less restrictive, which will permit the inclusion of this device as a special case.

2. Kakutani's theorem. For each positive integer $n$, let $\mathcal{B}_{n}$ be a (countably) additive class of subsets of a set $\Omega_{n}$, and let $m_{n}$ be a (countably additive) measure on $B_{n}$, such that $m_{n}\left(\Omega_{n}\right)=1$. Let $\Omega^{*}$ be the infinite product space $\Omega_{1} \times \Omega_{2} \times \cdots$ of all sequences $\omega^{*}=\left\{\omega_{n}\right\}$, $\omega_{n} \in \Omega_{n}(n=1,2, \cdots)$. Let $R^{*}$ be the family of all rectangular sets $R^{*}$,

$$
R^{*}=B_{1} \times B_{2} \times \cdots \times B_{k} \times \Omega_{k+1} \times \Omega_{k+2} \times \cdots,
$$

where $B_{i} \in B_{i}(i=1,2, \cdots, k ; 1,2, \cdots)$. Let $\mathbb{B}^{*}$ be the additive class of sets of $\Omega^{*}$ generated by $R^{*}$, and $m^{*}$ the measure $[1,2$; cf. 1949.

Presented to the Society, December 29, 1949; received by the editors March 21, 
also 3 $]^{1}$ on $\mathbb{B}^{*}$ such that $m^{*}\left(R^{*}\right)=\prod_{n=1}^{k} m_{n}\left(B_{n}\right)$.

The condition $m_{n}\left(\Omega_{n}\right)=1$ may be replaced by $0<\prod_{1}^{k} m_{n}\left(\Omega_{n}\right)<\infty$, a measure $m^{*}$ then being determined on $B^{*}$ such that $m^{*}\left(R^{*}\right)$ $=\prod_{1}^{k} m_{n}\left(B_{n}\right) \prod_{k+1}^{\infty} m_{n}\left(\Omega_{n}\right)$. It is sufficient to consider the measures $\mu_{n} \equiv m_{n} / m_{n}\left(\Omega_{n}\right)$, which satisfy $\mu_{n}\left(\Omega_{n}\right)=1$, and to define $m^{*}\left(B^{*}\right)$ $=\mu^{*}\left(B^{*}\right) \prod_{1}^{\infty} m_{n}\left(\Omega_{n}\right)$ for $B^{*} \in B^{*}$.

One may define similarly $\Omega_{k}^{*}=\Omega_{k} \times \Omega_{k+1} \times \cdots, R_{k}^{*}, B_{k}^{*}$, and $m_{k}^{*}(k=1,2, \cdots)$.

LEMMa 1. If

$$
E^{*}=\Omega_{1} \times \Omega_{2} \times \cdots \times \Omega_{k-1} \times E_{k}^{*}, \quad E_{k}^{*} \in \mathscr{B}_{k}^{*},
$$

then $E^{*} \in \mathcal{B}^{*}$, and $m^{*}\left(E^{*}\right)=m_{1}\left(\Omega_{1}\right) m_{2}\left(\Omega_{2}\right) \cdots m_{k-1}\left(\Omega_{k-1}\right) m_{k}^{*}\left(E_{k}^{*}\right)$ [4, p. 145].

Definition. Let $\boldsymbol{B}$ be an additive family of subsets of set $\Omega$ of elements $\omega$. Measures $m, m^{\prime}$ defined on $B$ are equivalent $\left(m \sim m^{\prime}\right)$ on $\mathscr{B}$ if each is absolutely continuous with respect to the other; that is, if $m(B)=0$ implies $m^{\prime}(B)=0$ for $B \in \mathcal{B}$, and conversely. It follows from a theorem of Radon-Nikodym (cf. S. Saks, Theory of the integral, 1937, p. 36) that there exists a $B$-measurable function $\phi(\omega)$ defined on $\Omega$ such that

$$
m^{\prime}(B)=\int_{B} \phi(\omega) d m(\omega) \quad \text { for } B \in \mathbb{B},
$$

and a function $\psi(\omega)$ such that $m(B)=\int_{B} \psi(\omega) d m^{\prime}(\omega)$, for $B \in \mathcal{B}$.

Let $m_{n}, m_{n}^{\prime}$ be two additive measures on $\mathcal{B}_{n}(n=1,2, \cdots \cdot)$.

TheOREM 1. (KaKUTANI's THEOREM [1]). If

$$
\begin{aligned}
m_{n} & \sim m_{n}^{\prime} & \text { on } \mathcal{B}_{n}, \\
m_{n}\left(\Omega_{n}\right) & =m_{n}^{\prime}\left(\Omega_{n}\right)=1, &
\end{aligned}
$$

then a necessary and sufficient condition that $m^{*} \sim m^{* \prime}$ on $\mathcal{B}^{*}$ is that

$$
\prod_{n=1}^{\infty} \rho_{n}>0 \text {, }
$$

where

$$
\rho_{n}=\rho\left(m_{n}, m_{n}^{\prime}\right)=\int_{\Omega}\left(\phi_{n}(\omega)\right)^{1 / 2} d m_{n}(\omega),
$$

and where $\phi_{n}(\omega)$ is such that

1 Numbers in brackets refer to the bibliography at the end of the paper. 


$$
m_{n}^{\prime}(B)=\int_{B} \phi_{n}(\omega) d m_{n}(\omega) \quad \text { for } B \in \mathcal{B}_{n}(n=1,2, \cdots) .
$$

An immediate consequence of Kakutani's theorem is the following lemma.

LEMma 2. If (1) holds $(n=1,2, \cdots)$,

$$
\begin{aligned}
m_{n}\left(\Omega_{n}\right) & \leqq 1, \quad m_{n}^{\prime}\left(\Omega_{n}\right) \leqq 1 \quad(n=1,2, \cdots), \\
\prod_{1}^{\infty} m_{n}\left(\Omega_{n}\right)>0, & \prod_{1}^{\infty} m_{n}^{\prime}\left(\Omega_{n}\right)>0,
\end{aligned}
$$

and (3) holds, then $m^{*} \sim m^{* \prime}$ on $\mathcal{B}^{*}$.

It is sufficient to apply Kakutani's theorem to the measures $\mu_{n}(B)=m_{n}(B) / m_{n}\left(\Omega_{n}\right)$ and $\mu_{n}^{\prime}(B)=m_{n}^{\prime}(B) / m_{n}^{\prime}\left(\Omega_{n}\right) \quad\left(B \in \mathcal{B}_{n}\right)$. For, then

$$
\rho\left(\mu_{n}, \mu_{n}^{\prime}\right)=\rho\left(m_{n}, m_{n}^{\prime}\right) /\left(m_{n}\left(\Omega_{n}\right) m_{n}^{\prime}\left(\Omega_{n}\right)\right)^{1 / 2},
$$

hence $\prod_{1}^{\infty} \rho\left(\mu_{n}, \mu_{n}^{\prime}\right)>0$ (note that by $\operatorname{Sch}$ warz' inequality $\rho\left(\mu_{n}, \mu_{n}^{\prime}\right)$ $\leqq 1$ ). Thus $\mu^{*} \sim \mu^{* \prime}$, and $m^{*} \sim m^{* \prime}$ (p. 410).

Let $\mathcal{F}^{*}$ denote the family of all sets of $\mathcal{B}^{*}$ invariant under transformations replacing at most a finite number of coordinates $\omega_{1}$, $\omega_{2}, \cdots, \omega_{k}$ of a point of the set by others from $\Omega_{1}, \Omega_{2}, \cdots, \Omega_{k}$ respectively. $\mathcal{F}^{*}$ is the family of all sets of $\mathcal{B}^{*}$ which, for every positive integer $k$, can be expressed in the form:

$$
\Omega_{1} \times \Omega_{2} \times \cdots \times \Omega_{k-1} \times B_{k}^{*} \quad\left(B_{k}^{*} \in \mathscr{B}_{k}^{*}\right) .
$$

Clearly $\mathcal{F}^{*}$ is an additive class of sets.

LEMMA 3. If $m_{n}\left(\Omega_{n}\right)=\mu_{n}\left(\Omega_{n}\right)=1(n=1,2, \cdots)$, and if there exists a positive integer $n_{0}$ such that $m_{n}=\mu_{n}$ for $n \geqq n_{0}$, then $m^{*}=\mu^{*}$ on $\mathcal{F}^{*}$.

For, then $m_{n_{0}}^{*}=\mu_{n_{0}}^{*}$; and since every set of $\mathcal{F}^{*}$ is of the form $\Omega_{1} \times \Omega_{2}$ $\times \cdots \times \Omega_{n_{0}-1} \times E_{n_{0}}^{*}$ for a set $E_{n_{0}}^{*} \in \mathcal{B}_{n_{0}}^{*}$, this is an immediate consequence of Lemma 1 .

Let a set $B_{n}$ be chosen from each family $\mathcal{B}_{n}(n=1,2, \cdots)$. Denote by $\mathcal{B}_{n} \cdot B_{n}(n=1,2, \cdots)$ the additive class of all sets of $\mathcal{B}_{n}$ contained in $B_{n}$. The following theorem assumes that Kakutani's conditions hold only on the subsets $B_{n}$ of $\Omega_{n}$, with a supplementary condition $(7)^{\prime}$ on the $B_{n}$, and states that the infinite product measures are equal on $\mathcal{F}^{*}$.

THEOREM 2. If there exists a positive integer $n_{0}$ such that

$$
m_{n} \sim m_{n}^{\prime}
$$

on $\mathcal{B}_{n} \cdot B_{n}$, 


$$
\begin{gathered}
m_{n}\left(\Omega_{n}\right)=m_{n}^{\prime}\left(\Omega_{n}\right)=1, \\
\prod \int_{B_{n}}\left(\phi_{n}(\omega)\right)^{1 / 2} d m_{n}(\omega)>0, \\
\prod m_{n}\left(B_{n}\right)>0, \quad \prod m_{n}^{\prime}\left(B_{n}\right)>0,
\end{gathered}
$$

all for $n \geqq n_{0}$, then $m^{*}=m^{* \prime}$ on $\mathcal{F}^{*}$.

It is no restriction to suppose $n_{0}=1$. For if $n_{0}>1$, let $\mu_{n}$ and $\mu_{n}^{\prime}$ be arbitrary measures satisfying $(1)^{\prime},(2)^{\prime}$, and $(7)^{\prime}$ for $n<n_{0}$ (for example $\mu_{n}(E)=\mu_{n}^{\prime}(E)=1$ if $E$ contains a fixed point $\omega_{n_{1}} \in B_{n}$, and $\mu_{n}(E)=\mu_{n}^{\prime}(E)=0$ if $\left.\omega_{n_{1}} \notin E\right)$, and $\mu_{n}(E)=m_{n}(E), \mu_{n}^{\prime}(E)=m_{n}^{\prime}(E)$ for $E \in \mathcal{B}_{n}\left(n \geqq n_{0}\right)$. Then assuming the theorem for $n_{0}=1, \mu^{*}=\mu^{* \prime}$ on $7^{*}$. Hence by Lemma $3, m^{*}=m^{* \prime}$ on $\mathcal{F}^{*}$.

Proof. Apply Lemma 2 to the sequences of measure spaces ${ }^{2}$ $\left\{B_{n}, \mathbb{B}_{n} \cdot B_{n}, m_{n}\right\}$ and $\left\{B_{n}, B_{n} \cdot B_{n}, m_{n}^{\prime}\right\}(n=k, k+1, \cdots)$ for each positive integer $k$. One finds $m_{k}^{*} \sim m_{k}^{* \prime}$ on $B_{k}^{*} \cdot B_{k}^{*}$, where $B_{k}^{*}=B_{k}$ $\times B_{k+1} \times \ldots$. Since every set of $\mathcal{F}^{*}$ is of the form $\Omega_{1} \times \Omega_{2} \times \ldots$ $\times \Omega_{k-1} \times E_{k}{ }^{*}$ for some $E_{k}^{*} \in B_{k}^{*}$, it follows from Lemma 1 that $m^{*} \sim m^{* \prime}$ on $\mathcal{F}^{*} \cdot A_{k}^{*}$, where

$$
\begin{aligned}
A_{k}^{*}= & \Omega_{1} \times \Omega_{2} \times \cdots \times \Omega_{k-1} \times B_{k} \times B_{k+1} \times \cdots \\
= & \bigcap_{j=1}^{\infty} \Omega_{1} \times \Omega_{2} \times \cdots \times \Omega_{k-1} \times B_{k} \times B_{k+1} \times \cdots \\
& \times B_{k+j} \times \Omega_{k+j+1} \times \cdots
\end{aligned}
$$

Let $A^{*}=\bigcup_{k=1}^{\infty} A_{k}^{*}=\lim _{k \rightarrow \infty} A_{k}^{*}$; and let $E^{*}$ be a set of $\mathcal{F}^{*} \cdot A^{*}$ such that $m^{*}\left(E^{*}\right)=0$. Then

$$
\begin{aligned}
E^{*} & =E^{*} \cdot A_{1}^{*} \cup E^{*} \cdot A_{2}^{*} \cup \cdots \\
m^{* \prime}\left(E^{*}\right) & \leqq m^{* \prime}\left(E^{*} \cdot A_{1}^{*}\right)+m^{* \prime}\left(E^{*} \cdot A_{2}^{*}\right)+\cdots \cdot
\end{aligned}
$$

But $m^{* \prime}\left(E^{*} \cdot A_{k}^{*}\right)=0(k=1,2, \cdots)$ since $m^{*} \sim m^{* \prime}$ on $\mathcal{F}^{*} \cdot A_{k}^{*}$. Hence $m^{* \prime}\left(E^{*}\right)=0$. Similarly $m^{*}\left(E^{*}\right)=0$ if $m^{* \prime}\left(E^{*}\right)=0, E^{*} \in \mathcal{F}^{*} \cdot A^{*}$. Thus $m^{*} \sim m^{* \prime}$ on $\mathcal{F}^{*} \cdot A^{*}$. But the measure $\left(m^{*}\right.$ or $\left.m^{* \prime}\right)$ of any set of $\mathcal{F}^{*}$ is either 0 or $1[4 ; 6$, p. 60$]$. Also

$$
m^{*}\left(A^{*}\right)=\lim _{k \rightarrow \infty} m^{*}\left(A_{k}^{*}\right)=\lim _{k \rightarrow \infty} \prod_{k}^{\infty} m_{n}\left(B_{n}\right)=1,
$$

and similarly $m^{* \prime}\left(A^{*}\right)=1$. Therefore the measure of any set of $\mathcal{F}^{*} \cdot A^{*}$

2 The triple $\{\Omega, \mathcal{B}, m\}$ denotes a measure space $\Omega$, and an additive class of sets $B$ on which a measure $m$ is defined. 
is either zero or one, so that $m^{*}=m^{* \prime}$ on $\mathcal{F}^{*} \cdot A^{*}$. Since also $m^{*}\left(E^{*}\right)$ $=m^{*}\left(E^{*} \cdot A^{*}\right)$ and $m^{* \prime}\left(E^{*}\right)=m^{* \prime}\left(E^{*} \cdot A^{*}\right)$ if $E^{*} \in \mathcal{F}^{*}, m^{*}=m^{* \prime}$ on $\mathcal{F}^{*}$. This completes the proof of Theorem 2 .

3. An application. Let $\left\{X_{n}\right\}$ and $\left\{Y_{n}\right\}$ be sequences of independent real-valued random variables with distribution functions $V_{n}$ and $W_{n}(n=1,2, \cdots)$, and corresponding linear measures $m_{n}$ and $m_{n}^{\prime}$. Let the sets $\Omega_{n}$ of the preceding discussion here all be $R_{1}$, the set of real numbers. $\Omega^{*}=R_{1} \times R_{1} \times \cdots$ is the space of sequences $\left(\omega_{1}, \omega_{2}, \cdots\right),-\infty<\omega_{n}<\infty(n=1,2, \cdots)$. Each of the families $B_{n}$ may be taken here as the family of Borel sets on $\Omega_{n}=R_{1}$. For $B_{n} \in B_{n}$, $m_{n}\left(B_{n}\right)$ represents the probability of $X_{n}$ belonging to $B_{n}$; for $B^{*} \in \mathcal{B}^{*}$, $m^{*}\left(B^{*}\right)$ represents $\operatorname{Pr}\left\{\left(X_{1}, X_{2}, \cdots\right) \in B^{*}\right\}$.

Given a limit theorem whose conclusion is a property (P) of $m^{*}$ on sets invariant under changes affecting at most a finite number of coordinates (that is, sets of $\mathfrak{f}^{*}$ ), Theorem 2 gives sufficient conditions that $m^{* \prime}$ have the same property. That is, Theorem 2 gives conditions sufficient in order that $\left\{Y_{n}\right\}$ may satisfy the limit theorem if $\left\{X_{n}\right\}$ does, and in this way extends any known conditions sufficient for the limit theorem.

Let us refer to such a limit theorem as a limit theorem $\left(\mathrm{P} ; m^{*}, \mathfrak{f}^{*}\right)$. Then as it applies to sequences of independent random variables, Theorem 2 becomes:

THEOREM 2'. If there exist a positive integer $n_{0}$ and sets $B_{n} \in B_{n}$ $\left(n \geqq n_{0}\right)$ such that

(1)' $\operatorname{Pr}\left\{X_{n} \in E_{n}\right\}=0$ if and only if $\operatorname{Pr}\left\{Y_{n} \in E_{n}\right\}=0$

$$
\text { for } E_{n} \in \mathcal{B}_{n} \cdot B_{n} \text {, }
$$

(3)' $\Pi \int_{B_{n}}\left(\phi_{n}(\omega)\right)^{1 / 2} d m_{n}(\omega)>0$,

(7)' $\Pi \operatorname{Pr}\left\{X_{n} \in B_{n}\right\}>0, \quad \prod \operatorname{Pr}\left\{Y_{n} \in B_{n}\right\}>0$

(or equivalently, $\sum \operatorname{Pr}\left\{X_{n} \notin B_{n}\right\}<\infty, \sum \operatorname{Pr}\left\{Y_{n} \notin B_{n}\right\}<\infty$ )

for all $n \geqq n_{0}$, then a necessary and sufficient condition that $\left\{Y_{n}\right\}$ obey a limit theorem $\left(\mathrm{P} ; m^{* \prime}, \mathcal{F}^{*}\right)$ is that $\left\{X_{n}\right\}$ obey it.

Many well known limit theorems are of this type. The strong law of large numbers, the law of the iterated logarithm, and Kolmogoroff's theorem on the probability of convergence of sequences of independent random variables are examples. The last named is a limit theorem $\left(\mathrm{P}_{1} ; m^{*}, \mathcal{F}^{*}\right)$, where $\left(\mathrm{P}_{1}\right)$ is the property: 
$\left(\mathrm{P}_{1}\right) \quad m^{*}\left(E_{1}^{*}\right)=1$, where $E_{1}^{*}=\left\{\sum_{1}^{\infty} X_{n}\right.$ converges $\} ;$

that is, $E_{1}^{*}$ is the set of points $\left(\omega_{1}, \omega_{2}, \cdots\right)$ of $\Omega^{*}$ such that $\sum_{1}^{\infty} \omega_{n}$ converges. Evidently $E_{1}^{*} \in \mathcal{F}^{*}$. The law of the iterated logarithm is a limit theorem $\left(\mathrm{P}_{2}: m^{*}, \mathcal{F}^{*}\right)$, where $\left(\mathrm{P}_{2}\right)$ is the property:

$$
\begin{gathered}
m^{*}\left(E_{2}^{*}\right)=1, \\
\text { where } E_{2}^{*}=\left\{\limsup _{n \rightarrow \infty} \sum_{1}^{n} X_{k} /\left[s_{n}\left(2 \log \log s_{n}\right)^{1 / 2}\right]=1\right\} .
\end{gathered}
$$

Here $s_{n}$ denotes the sum of the second moments about the mean (the mean is here taken to be zero) of the first $n$ random variables (cf. [5, p. 823]). Again $E_{2}^{*} \in \mathcal{F}^{*}$, if $s_{n} \rightarrow \infty$. The strong law of large numbers is a limit theorem $\left(\mathrm{P}_{3} ; m^{*}, \mathcal{F}^{*}\right)$, where $\mathrm{P}_{3}$ is the property:

$$
m^{*}\left(E_{3}^{*}\right)=1, \quad \text { whère } \quad E_{3}=\left\{1 / n \sum_{1}^{n} X_{k}=0\right\}
$$

(here again the mean of $X_{k}$ is assumed to be zero). Clearly $E_{3}^{*} \in \mathcal{F}^{*}$.

Theorem $2^{\prime}$ contains the following standard device. Let $\left\{Y_{n}\right\}$ be a sequence of independent random variables, and $\left\{B_{n}\right\}$ a sequence of sets $\left(B_{n} \subset \Omega_{n}, B_{n} \in B_{n}\right)$ such that $\sum_{1}^{\infty} \operatorname{Pr}\left\{Y_{n} \notin B_{n}\right\}<\infty$. Define the sequence of independent random variables $\left\{X_{n}\right\}$ such that $X_{n}=Y_{n}$ on $B_{n}$, that is, so that for each positive integer $n$ and for each $E \in \mathcal{B}_{n}$ - $B_{n}, m_{n}(E)=m_{n}^{\prime}(E)$. Then, if $\left\{X_{n}\right\}$ obeys a limit theorem (P; $\left.m^{*}, \mathcal{F}^{*}\right)$, so does the sequence $\left\{Y_{n}\right\}$. For then $\phi_{n}(\omega)=1$ on $B_{n}$, and also $\operatorname{Pr}\left\{X_{n} \notin B_{n}\right\}=1-\operatorname{Pr}\left\{X_{n} \in B_{n}\right\}=1-\operatorname{Pr}\left\{Y_{n} \in B_{n}\right\}=\operatorname{Pr}\left\{Y_{n}\right.$ $\left.\notin B_{n}\right\}$, hence the hypotheses of Theorem 2' are satisfied.

\section{BIBLIOGRAPHY}

1. Shizuo Kakutani, On equivalence of infinite product measures, Ann. of Math. (2) vol. 49 (1948) pp. 214-224.

2. - Notes on infinite product measure spaces, I, Proc. Imp. Acad. Tokyo vol. 19 (1943) pp. 148-151.

3. Z. Lomnicki and S. Ulam, Sur la theorie de la mesure dans les espaces combinatoires et son application au calcul des probabilités. I: Variables indépendantes, Fund. Math. vol. 23 (1934) pp. 237-278.

4. C. Visser, Law of nought or one in the theory of probability, Studia Mathematica vol. 7 (1938) pp. 143-149.

5. W. Feller, The fundamental limit theorems in probability, Bull. Amer. Math. Soc. vol. 51 (1945) pp. 800-832.

6. A. Kolmogoroff, Grundbegriffe der wahrscheinlichkeitsrechnung, Ergebnisse der Mathematik und ihre Grenzgebiete, vol. 2, no. 3, Berlin, 1933.

The Rice Institute 